\title{
Defining Disparities in Cochlear Implantation through the Social Determinants of Health
}

\author{
Marissa Schuh, M.P.H. ${ }^{1}$ and Matthew L. Bush, M.D., Ph.D., M.B.A. ${ }^{1}$
}

\section{ABSTRACT}

Hearing loss is a global public health problem with high prevalence and profound impacts on health. Cochlear implantation (CI) is a well-established evidence-based treatment for hearing loss; however, there are significant disparities in utilization, access, and clinical outcomes among different populations. While variations in CI outcomes are influenced by innate biological differences, a wide array of social, environmental, and economic factors significantly impact optimal outcomes. These differences in hearing health are rooted in inequities of health-related socioeconomic resources. To define disparities and advance equity in CI, there is a pressing need to understand and target these social factors that influence equitable outcomes, access, and utilization. These factors can be categorized according to the widely accepted framework of social determinants of health, which include the following domains: healthcare access/quality, education access/quality, social and community context, economic stability, and neighborhood and physical environment. This article defines these domains in the context of CI and examines the published research and the gaps in research of each of these domains. Further consideration is given to how these factors can influence equity in CI and how to incorporate this information in the evaluation and management of patients receiving cochlear implants.

KEYWORDS: cochlear implants, hearing, social determinants of health, equity, disparity

An estimated 1.5 billion people across the globe experience hearing loss and over 430 million are candidates for intervention. ${ }^{1}$ Hearing loss has profound impacts on communica- tion, education, socialization, cognition, and overall well-being across the lifespan. ${ }^{2}$ The prevalence of hearing loss and the need for hearing healthcare is expected to continue to
${ }^{1}$ Department of Otolaryngology - Head and Neck Surgery, University of Kentucky Medical Center, Lexington, Kentucky.

Address for correspondence: Matthew L. Bush, M.D., Ph.D., M.B.A., Department of Otolaryngology - Head and Neck Surgery, University of Kentucky Medical Center, 740 South Limestone, E300E, Lexington, KY 40536-0284 (email: matthew.bush@uky.edu).
Barriers to Cochlear Implant Access: Acknowledging the Challenges, Changing the Future; Guest Editor, Sarah Sydlowski, Au.D., Ph.D., M.B.A.

Semin Hear 2021;42:321-330. C 2021. Thieme. All rights reserved. Thieme Medical Publishers, Inc., 333 Seventh Avenue, 18th Floor, New York, NY 10001, USA DOI: https://doi.org/10.1055/s-0041-1739282.

ISSN 0734-0451. 
rise over the next quarter century. Underserved populations face an even higher prevalence and impact of hearing loss., Evidence-based treatments for hearing loss, such as cochlear implants, can significantly improve hearing-related outcomes and overall quality of life ${ }^{5}$; however, access to and utilization of such devices is neither uniform nor equitable. In spite of this expanding global public health problem, the utilization of hearing devices, including cochlear implants, across the world is less than $15 \%{ }^{6}$ and there are significant discrepancies in equitable hearing healthcare access and utilization among different populations. Biological and clinical differences involved in the pathophysiology and manifestation of hearing loss certainly influence outcomes following CI; however, a wide variety of social factors are at play as well. Even when care is accessed, clinical outcomes of cochlear implant recipients may vary widely among different populations ${ }^{7}$ and there is a pressing need to understand and address, in a broader sense, the social factors that influence equity in CI access, utilization, and outcomes. Pursuing equity is an ethical priority as many inequities in healthcare are rooted in discriminatory practices and racism. ${ }^{8}$ Furthermore, promoting equity has economic implications, as health inequities result in billions of lost dollars each year due to increased medical costs and lost productivity. ${ }^{9}$

Health-influencing social factors are categorized according to the widely accepted framework of social determinants of health, which is defined by where people live, work, and play that can either directly or indirectly influence their health and overall quality of life. ${ }^{10}$ Additionally, these factors play a role in the severity and the distribution of health disparities. Defining, describing, and addressing the social determinants of health is a priority area for the World Health Organization (WHO) to tackle health inequities, such as $\mathrm{CI}$ access differences between different nations. ${ }^{11,12}$ The social determinants of health framework can be divided into five domains which include the following: healthcare access and quality, education access and quality, social and community context, economic stability, and neighborhood and physi- cal environment. ${ }^{10,13}$ The first domain concentrates accessibility and utilization of healthcare services among patients as well as their health knowledge. For example, this could include type and amount of insurance coverage, provider availability and accessibility, health literacy, and the quality of care. The second domain of the social determinants of health focuses on the accessibility and quality of education and its effects on health outcomes. Examples of factors from this domain include level of education, access to vocational training, parental educational attainment, access to early childhood education, and school-based rehabilitation services. The next domain, social and community context, evaluates the social conditions and connections of individuals and how they impact one's overall health and well-being. This domain incorporates social support systems and networks, community engagement, social integration, and cultural/racial/ethnic social identity. The impact of discrimination based on race, ethnicity, gender, or sexual orientation would be assessed within this domain. Economic stability represents the fourth domain and encompasses individual financial and material resources and how they impact health. Some factors under this domain include employment, income, poverty, debt, or expenses, as well as food and housing security. The final domain of this framework is the neighborhood and physical environment, which involves the relationship between where people live and the impact on their health and well-being. For example, this domain would connect health with the safety and quality of housing, accessibility and utilization of transportation, water and air quality, neighborhood crime, and rurality of household location. These five domains represent a comprehensive framework that can be used to evaluate inequities in any aspect of health or healthcare and these factors, either positively or negatively, influence the health of every single person who is eligible for or who receives a cochlear implant. The objective of this article is to define disparities in CI through the lens of the social determinants and identify targets and methods to promote equity in patients who would benefit from CI. 


\section{SOCIAL DETERMINANTS OF HEALTH}

\section{Healthcare Access and Quality}

Utilization of and access to quality hearing healthcare services influences overall health and hearing-related outcomes for adults and children. Considering the global underutilization of hearing devices, access to care is a critical issue for CI specialists and researchers to evaluate and address. Access to CI care is inherently complex, as it requires transdisciplinary longterm care which is provided by different types of specialists potentially in different locations over the course of a patient's life. A patient's access to any type of healthcare, along with CI care, can be restricted by a variety of different geographic and socioeconomic position (SEP) factors. The geographic location of a patient's household affects a patient's access to CI care. Certainly, $\mathrm{CI}$ is not an accessible hearing loss treatment option for every country; however, access to CI care can be exceedingly limited in rural or remote regions of any country. Limitations in rural hearing healthcare access is a pressing issue, as rural adults face a greater burden of age-related hearing loss compared with urban areas. ${ }^{14,15}$ Children residing in rural areas are also more likely to experience delays with every aspect of hearing healthcare including diagnosis of hearing loss, hearing aid amplification, and cochlear implantation. ${ }^{16-18}$ Delays in cochlear implantation have negative impacts on speech and language development in children. ${ }^{19}$ Furthermore, children from rural areas face greater difficulty in accessing rehabilitation services post-CI surgery due to a lack of local providers thereby increasing costs and travel for families. ${ }^{7}$ Similar findings in adult populations demonstrate that rural adults with hearing loss are delayed in CI compared with their urban peers, which is also related to lack of local access and greater travel distances to cochlear implant centers. $^{20}$

Insurance coverage or lack thereof represents a key factor influencing if and where healthcare is accessed across a wide range of medical disciplines. Insurance status and type of insurance has been used as a proxy for SEP but may be influenced by various factors including income, employment, or race and ethnicity.
There is evidence that non-white patients and minority ethnic groups are more likely to have public insurance coverage instead of private insurance coverage compared with white patients. ${ }^{21}$ While cochlear implantation is typically covered for children by Medicaid and most private insurance carriers in the United States, the same is not true for adults. Among adults who are insured through Medicaid, cochlear implant coverage is optional depending on the state's criteria. Currently, only approximately $60 \%$ of the States offer Medicaid coverage for cochlear implantation in adults. ${ }^{22}$ Even if CI Medicaid coverage is available, the quality of care that is delivered may be negatively impacted by barriers in obtaining upgraded or replacement equipment, poor reimbursement, limitations in locations of care, limitations in the number of covered appointments, and difficulty in authorization for care. ${ }^{22}$ In some states, a patient who received a Medicaid-covered cochlear implant as a child may grow out of service eligibility to receive necessary care once they transfer to adult Medicaid coverage. ${ }^{22}$ Furthermore, there is evidence that Medicaid patients are 50\% less likely to receive sequential bilateral cochlear implants compared with those who were privately insured. $^{23}$ Furthermore, patients covered by Medicaid were five times more likely to experience post-surgery complications and less likely to comply with follow-up appointments compared with privately insured patients. ${ }^{7,23}$ From a clinic perspective, one major challenge of Medicaid coverage for $\mathrm{CI}$ is the low reimbursement rate throughout the continuum of care from surgery to rehabilitation and may lead to limiting access for these patients. ${ }^{22}$

While the racial and ethnic health disparities may be influenced by various determinants, there are long-standing differences in access to and quality of healthcare services in a wide range of health conditions based on race and ethnicity. This racial and ethnic disparity has been demonstrated in adult CI research as patients of non-white racial groups are less likely to undergo CI surgery for cochlear implants despite being eligible candidates. ${ }^{24}$ Similarly, children who are from non-white racial groups are delayed in CI regardless of their insurance type and coverage and are less 
likely to be implanted before the age of 2 years compared with white children. ${ }^{25}$ This racial/ ethnic inequity is critical to address considering the developmental implications of early implantation among pediatric patients. In addition to access to care, unconscious bias or discrimination based on race and/or ethnicity from medical professionals may contribute and play a role in the quality of care that patients receive. ${ }^{21}$ Understanding how patients access and utilize hearing healthcare services and the barriers to that care will provide insight and possible solutions on how to increase equitable healthcare for hearing loss.

\section{Education Access and Quality}

Educational access and attainment influences health through various pathways. Education attainment intersects with other social determinants of health through its impact on income and higher wages which in turn affects access to healthy food, safe living environments, and insurance coverage. ${ }^{26}$ Therefore, it is not surprising that there is a correlation between educational attainment and health outcomes in which lower education attainment is associated with poorer health outcomes. Adult CI outcomes have been directly correlated with educational attainment levels. In the area of pediatric CI, parent's educational level has been associated with the utilization of hearing healthcare and with the speech development outcomes of their child. Lower parental education attainment may predict underutilization of speech therapy following pediatric CI. ${ }^{27}$

Health literacy is directly connected to educational attainment, which in concert influences behaviors that can promote health. Individuals with lower health literacy are less likely to obtain necessary care and may report more difficulty with finding providers compared with those with greater health literacy. ${ }^{28}$ This inability to understand the language and systems of healthcare plays a role in hearing healthcare, as patients with low health literacy may not be aware of potential treatments of hearing loss and may not seek out hearing healthcare services. ${ }^{29}$ Consequently, the delay in pursuing hearing services and their duration of hearing loss may influence their clinical outcomes. ${ }^{29}$
Health literacy also influences the cost of care, as there is a strong correlation with lower health literacy and higher medical expenses among patients with hearing loss. ${ }^{30,31}$ English-fluency, while not directly connected to health literacy, strongly influences communication between patients and providers within the United States and may influence overall health outcomes. There is a link between language barriers, patient satisfaction, care adherence, and utilization of healthcare services. ${ }^{32}$

The language barriers between parents of deaf or hard of hearing children may influence the timing of hearing healthcare service delivery and therefore hearing outcomes of that child. Addressing the educational needs for patients needing cochlear implantation is a daunting issue; however, there are ample opportunities to promote health literacy of individual patients and the public regarding hearing loss and the evidence-based treatment options. This domain begs for innovation and intervention development.

\section{Social and Community Context}

Social support through systems and relationships as well as interactions with individuals and community members can also impact health outcomes. ${ }^{33}$ Relationship support enhances overall health and decreases mortality, while the lack of social support and connectedness can precipitate adverse health outcomes. In hearing healthcare, the presence of a strong social support system impacts the timing and delivery of hearing healthcare. ${ }^{34}$ Moreover, cultural differences and community perspectives on hearing loss can shape how individuals perceive their own hearing loss and the choices they make regarding treatment. ${ }^{35}$ Social support systems also influence adherence with wearing hearing devices. Children lacking support and behavioral reinforcement at school and within the home are less adherent with wearing their cochlear implants. ${ }^{36}$ Similarly, elderly adult patients who were nonadherent with cochlear implant usage also lack social support. ${ }^{37}$ Overall, social support systems can influence hearing healthcare from the onset of the condition to the utilization of hearing devices and services. This social determinants of health (SDH) 
domain forms the basis for comprehensive cochlear implant teams that incorporate key social support members and systems into cochlear implant counseling and care delivery.

In addition to support systems, social identity can affect how individuals seek and utilize care, thereby affecting health outcomes. Social identify is defined as an individual's sense of who they are in relation to their group or community. ${ }^{38}$ Children and adults who are deaf or hard of hearing may experience dissonance in defining their social identity when coexisting among hearing peers and family members. School-aged children with cochlear implants may face challenges in developing close relationships with their peers and this may predispose them to mental health problems. ${ }^{39}$ Furthermore, adults with hearing loss report marginalization and social isolation due to their hearing loss, which may influence the development of depression in these individua1s. ${ }^{40,41}$ In some cases, cochlear implant users may face an identity crisis between the hearing community and the deaf community. ${ }^{42}$ Related to social identity, stigma around deafness and hearing loss heavily influences hearing health behaviors and outcomes. Stigma regarding hearing loss stemming from social contexts has been associated with poor mental health and overall decreased quality of life. ${ }^{42,43} \mathrm{Co}^{-}$ chlear implant users who identify with the deaf community may perceive more discrimination than those who identify with the hearing world. ${ }^{44}$ In addition to the discrimination based on hearing status, cochlear implant users from non-white racial and ethnic groups experience collateral discrimination. ${ }^{44}$ Discriminatory policies and practices based on social contexts or identity influence not only individual health and healthcare but also other public health due to the impact on other social determinants of health, such as employment, housing, and education. ${ }^{21}$

\section{Economic Stability}

Economic stability can influence health outcomes both individually and within a household. Elements of economic stability include employment and income, SEP, as well as food and housing security. These elements can im- pact one's health through various avenues. First, employment directly affects a household's economic stability by providing not only income but also insurance coverage and other benefits. An employed individual or household could still have a low SEP and thereby limited access and availability of information and resources to manage health conditions, such as hearing loss. Patient or families with significant economic instability may not prioritize health and healthcare services. Even when financial resources are made available to lower SEP families, those resources are often underutilized. ${ }^{45}$ Families of deaf or hard of hearing children or adults who live closer to the poverty line are less likely to utilize a wide range of medical services, including hearing healthcare. ${ }^{46,47}$ Children from lower-income households are less likely to receive a cochlear implant, in spite of having a higher prevalence of hearing loss compared with those from higher-income families. ${ }^{48}$ Moreover, lower family SEP has been associated with delayed cochlear implantation among children younger than 3 years. ${ }^{49}$ Even after implantation, pervasive disparities remain for CI users of lowincome households. Patients from a lower SEP background are more likely to experience postoperative complications, to be nonadherent with follow-up appointments, and to receive only unilateral and not bilateral sequential $\mathrm{CI} .^{23}$ Furthermore, those same patients experience poorer speech and language outcomes post-CI compared with those of a higher SEP background. ${ }^{50}$ Similarly, adult patients of lower SEP demonstrate poorer speech perception gains after cochlear implantation. ${ }^{47}$

Families of low economic stability also face other challenges that affect health outcomes, such as limited food and housing security. Families who live close to the poverty line are more likely to face food insecurity and have limited access to healthy foods. This is amplified for non-white racial and ethnic groups. ${ }^{51}$ Food insecurity is also more prevalent among those with hearing loss than those with normal hearing. ${ }^{52}$ It is intuitive that individuals with lower incomes are also more likely to experience homelessness; however, racial and/or ethnic status influences homeless as demonstrated by the estimate that $40 \%$ of the homeless population are African American. ${ }^{53}$ This is pertinent 
because homeless adults tend to have a higher prevalence of chronic diseases, including hearing loss, and experience poorer access to care due to barriers such as lack of health insurance, prioritization of other physical needs, and discriminatory practices by the healthcare system toward this population. ${ }^{54}$ Furthermore, awareness regarding the types and availability of hearing-related resources is poor among homeless adults. ${ }^{55}$ In spite of the availability of cutting edge technology, the hearing healthcare field faces a great dilemma in engaging a diverse population with a wide range of socioeconomic resources and delivering affordable care in a culturally acceptable way.

\section{Neighborhood and Physical Environment}

While homelessness represents extreme challenges for health, the location and quality of housing can also influence one's health either positively or negatively. There is a direct relationship between home and neighborhood improvements and overall health within a community. ${ }^{56}$ Poor drinking water, mold, lead exposure, pests, environmental exposures, secondhand smoke exposure, and inadequate heating/ cooling are housing condition factors that can negatively affect health. While this SDH domain may seem indirectly related to cochlear implantation outcomes and care, it is feasible that environmental exposures, such as high levels of noise, or overall poor living conditions could increase the chances of developing chronic diseases or infectious diseases and could thereby influence the development or progression of hearing loss. It is valuable to consider this domain in relation to the other domains and the overall health and ability of cochlear implant recipients to pursue a healthy lifestyle. The established neighborhood conditions such as types and access to schools, employment opportunities, crime rates, food access, and healthcare infrastructure are all aspects of neighborhoods where cochlear implant recipients live and each of these factors impact the health of these patients. Again, racial and ethnic inequities are long-standing persistent issues based on neighborhood resources and stability as non-white racial ethnic groups make up a higher population percentage in neighborhoods with lower resources that could promote health. Consequently, there is a shortage of hearing healthcare specialists in these same resource-poor neighborhoods. For example, transportation to healthcare clinics may be lacking in resource-poor neighbors. This is significant as decreased access to personal or public transportation, which is more common among non-white racial and ethnic groups, directly affects utilization of healthcare and thereby overall health. ${ }^{57,58}$ When hearing specialists neither live nor physically work within these neighborhoods, those community members lack a sense of trust, connection, and engagement with overall hearing healthcare. This physical neighborhood disconnect with cochlear implant centers is difficult to overcome, but opportunities are present to proactively engage key stakeholders and community members to be a part of making meaningful linkages with hearing healthcare teams. ${ }^{59}$

\section{DISCUSSION}

This review of the social determinants of health serves to promote awareness of how these factors can influence cochlear implantation and complex hearing healthcare access, utilization, and delivery. While this explanation and discussion of the factors within each domain above is not exhaustive, it provides a framework to inform a more comprehensive understanding of the factors beyond the cochlea and underlying biology that influence hearing health and healthcare. It is critical for providers and researchers to recognize how the social determinants of health influence equity within hearing healthcare and explore unstudied aspects of this framework. Furthermore, this framework can provide mechanistic explanations for variations in outcomes following cochlear implantation. The social determinants of health can provide critical information and insight into the factors that influence hearing health outcomes and each of these domains can be targeted to promote optimal CI-related outcomes for all.

It is difficult to address SDH-rooted disparities without measuring SDH factors and these measures are not part of cochlear implant candidacy evaluations. Although it may not be a 
part of traditional evaluation of hearing healthcare specialists, providers can utilize a wide array of validated assessment tools to collect social determinant health data on cochlear implant candidates and users. For example, the Centers for Medicare and Medicaid Services Accountable Health Communities created the Health-Related Social Needs Screening Tool. ${ }^{60}$ This 10-item questionnaire gathers data on Medicare and Medicaid patients' social needs related to the different five social determinants of health domains to inform clinical decision-making and potentially impact health outcomes and healthcare costs. The National Association of Communication Health Centers also developed a tool called the "Protocol for Responding to and Assessing Patients' Assets, Risk and Experiences (PRAPARE)." ${ }^{61}$ This 21-item tool is a much more comprehensive set of measures that are rooted in public health research and informed by stakeholder input. This inventory focuses on areas where action can be taken to address inequities from a public health perspective. This measure could easily be incorporated into CI candidacy evaluations and could inform CI teams about potential factors that could influence outcomes. A third tool that is publicly available is the Social Needs Screening Tool designed by the American Academy of Family Physicians as part of their EveryOne Project. ${ }^{62}$ This 15 -item tool is rooted in clinical practice and has been used to identify basic barriers to care and underlying social needs facing patients. This information can be used to mobilize resources to address those needs in a timely fashion. Further study is needed in this area regarding the value and utility of this information for CI teams and how these data may correlate with cochlear implantation outcomes and if targeting these SDH domains will influence equity in cochlear implantation. In addition to these different quantitative measures of social determinants of health, hearingrelated research would benefit from incorporation of mixed methodology which utilizes qualitative methods to better define and describe the complex and interconnected nature of these domains on health. To increase the equitable utilization of cochlear implants and maximize hearing health for all, leaders in cochlear implantation are encouraged to con- sider and measure factors from each domain of the social determinants of health in their patients and develop informed targeted interventions and programs that address those needs. Comprehensive CI care extends far beyond the cochlea and incorporating social determinants of health information into how care is given and to whom care is given will promote health equity for diverse populations and communities.

\section{CONCLUSION}

The five domains of the social determinants of health impact hearing health and healthcare in a wide array of mechanisms over the lifespan. Factors from these domains influence how and when patients receive cochlear implants and can be used in part to explain varying outcomes following cochlear implantation. While collection of SDH data has not been a core component of CI candidacy or postoperative outcome measures, there is a need to better understand how these social determinants of health affect patients' access and utilization of CI-related services. This information can be used by CI teams to develop and implement interventions, programs, and policies that address disparities affecting their patients. Several validated tools can be used to systematically evaluate health-influencing factors from these five domains. Equity in cochlear implantation access, utilization, and outcomes is dependent on whether this information is considered and used.

\section{FUNDING}

This work was supported by the National Institute of Deafness and Other Communication Disorders (R01DC017770; M.L.B.). The funding organization had no input into the content of this manuscript. M.L.B. is a consultant for MED-EL and Stryker and has received research funding from Advanced Bionics (unrelated to this research).

\section{CONFLICTS OF INTEREST}

There are no conflicts of interests with the content of this manuscript. The authors have no other financial relationships or conflicts of 
interest to disclose pertaining to the manuscript.

\section{REFERENCES}

1. World report on hearing. Geneva: World Health Organization; 2021. License: CC BY-NC-SA 3.0 IGO

2. Kramer SE, Kapteyn TS, Kuik DJ, Deeg DJ. The association of hearing impairment and chronic diseases with psychosocial health status in older age. J Aging Health 2002;14(01):122-137

3. Mehra S, Eavey RD, Keamy DG Jr. The epidemiology of hearing impairment in the United States: newborns, children, and adolescents. Otolaryngol Head Neck Surg 2009;140(04):461-472

4. Merchant JA, Stromquist AM, Kelly KM, Zwerling C, Reynolds SJ, Burmeister LF. Chronic disease and injury in an agricultural county: the Keokuk County Rural Health Cohort Study. J Rural Health 2002;18(04):521-535

5. Gaylor JM, Raman G, Chung Met al.. Cochlear implantation in adults: a systematic review and meta-analysis. JAMA Otolaryngol Head Neck Surg 2013;139(03):265-272

6. World Health Organization. Assistive devices/ technologies: What WHO is doing. World Health Organization Accessed June 2021 at: http://www. who.int/disabilities/technology/activities/en/

7. Noblitt B, Alfonso KP, Adkins M, Bush ML. Barriers to rehabilitation care in pediatric cochlear implant recipients. Otol Neurotol 2018;39(05): e307-e313

8. Lu MC, Halfon N. Racial and ethnic disparities in birth outcomes: a life-course perspective. Matern Child Health J 2003;7(01):13-30

9. Mitchell RJ, Bates P. Measuring health-related productivity loss. Popul Health Manag 2011;14 (02):93-98

10. Centers for Disease Control and Prevention. Social Determinants of Health: Know What Affects Health. Accessed June 10, 2021 at: https://www. cdc.gov/socialdeterminants/about.htmlhttps:// www.who.int/health-topics/social-determinantsof-health\#tab=tab_1

11. Raine C, Atkinson H, Strachan DR, Martin JM. Access to cochlear implants: time to reflect. Cochlear Implants Int 2016;17(Suppl 1):42-46

12. Fagan JJ, Tarabichi M. Cochlear implants in developing countries: practical and ethical considerations. Curr Opin Otolaryngol Head Neck Surg 2018;26(03):188-189

13. World Health Organization. Social determinants of health. World Health Organization Access June 2021 at: https:/www.who.int/health-topics/ social-determinants-of-health\#tab=tab_1
14. Yong M, Willink A, McMahon Cet al.. Access to adults' hearing aids: policies and technologies used in eight countries. Bull World Health Organ 2019; 97(10):699-710

15. Hay-McCutcheon MJ, Threadgill M, Yang X, Phillips F. Access to hearing health care, geographical residency, and quality of life in adults with and without hearing loss. J Am Acad Audiol 2020;31 (07):485-495

16. Bush ML, Burton M, Loan A, Shinn JB. Timing discrepancies of early intervention hearing services in urban and rural cochlear implant recipients. Otol Neurotol 2013;34(09):1630-1635

17. Bush ML, Osetinsky M, Shinn JBet al.. Assessment of Appalachian region pediatric hearing healthcare disparities and delays. Laryngoscope 2014;124(07):1713-1717

18. Bush ML, Bianchi K, Lester Cet al.. Delays in diagnosis of congenital hearing loss in rural children. J Pediatr 2014;164(02):393-397

19. Yoshinaga-Itano C, Sedey AL, Wiggin M, Mason CA. Language outcomes improved through early hearing detection and earlier cochlear implantation. Otol Neurotol 2018;39(10):1256-1263

20. Hixon B, Chan S, Adkins M, Shinn JB, Bush ML. Timing and impact of hearing healthcare in adult cochlear implant recipients: a rural-urban comparison. Otol Neurotol 2016;37(09):1320-1324

21. Bulatao RA, Anderson NB. National Research Council(US) Panel on Race, Ethnicity, and Health in Later Life, eds. Understanding Racial and Ethnic Differences in Health in Late Life: A Research Agenda. Washington, DC: National Academies Press; 2004

22. Sorkin DL. Impact of Medicaid on cochlear implant access. Otol Neurotol 2019;40(03): e336-e341

23. Chang DT, Ko AB, Murray GS, Arnold JE, Megerian CA. Lack of financial barriers to pediatric cochlear implantation: impact of socioeconomic status on access and outcomes. Arch Otolaryngol Head Neck Surg 2010;136(07):648-657

24. Tolisano AM, Schauwecker N, Baumgart Bet al.. Identifying disadvantaged groups for cochlear implantation: demographics from a large cochlear implant program. Ann Otol Rhinol Laryngol 2020;129(04):347-354

25. Liu X, Rosa-Lugo LI, Cosby JL, Pritchett CV. Racial and insurance inequalities in access to early pediatric cochlear implantation. Otolaryngol Head Neck Surg 2021;164(03):667-674

26. Hahn RA, Truman BI. Education improves public health and promotes health equity. Int J Health Serv 2015;45(04):657-678

27. Sharma SD, Cushing SL, Papsin BC, Gordon KA. Hearing and speech benefits of cochlear implantation in children: a review of the literature. Int J Pediatr Otorhinolaryngol 2020;133:109984 
28. Levy H, Janke A. Health literacy and access to care. J Health Commun 2016; 21 Suppl 1(Suppl)43-50

29. Tran ED, Vaisbuch Y, Qian ZJ, Fitzgerald MB, Megwalu UC. Health literacy and hearing healthcare use. Laryngoscope 2021;131(05):E1688-E1694

30. Tolisano AM, Fang LB, Isaacson B, Kutz JW Jr, Hunter JB. Can you hear me now? The impact of hearing loss on patient health literacy. Otol Neurotol 2020;41(08):1027-1032

31. Wells TS, Rush SR, Nickels LD, Wu L, Bhattarai GR, Yeh CS. Limited health literacy and hearing loss among older adults. Health Lit Res Pract 2020; 4(02):e129-e137

32. Timmins CL. The impact of language barriers on the health care of Latinos in the United States: a review of the literature and guidelines for practice. J Midwifery Womens Health 2002;47(02):80-96

33. Holt-Lunstad J, Smith TB, Layton JB. Social relationships and mortality risk: a meta-analytic review. PLoS Med 2010;7(07):e1000316

34. Mahoney CF, Stephens SD, Cadge BA. Who prompts patients to consult about hearing loss? Br J Audiol 1996;30(03):153-158

35. Laplante-Lévesque A, Hickson L, Worrall L. Factors influencing rehabilitation decisions of adults with acquired hearing impairment. Int J Audiol 2010;49(07):497-507

36. Archbold SM, Nikolopoulos TP, Lloyd-Richmond H. Long-term use of cochlear implant systems in paediatric recipients and factors contributing to non-use. Cochlear Implants Int 2009;10 (01):25-40

37. Lundin K, Näsvall A, Köbler S, Linde G, RaskAndersen H. Cochlear implantation in the elderly. Cochlear Implants Int 2013;14(02):92-97

38. Tajfel H, Turner JC, Austin WG, Worchel S. An integrative theory of intergroup conflict. Organizational Identity1979:56-65

39. Anmyr L, Olsson M, Freijd A, Larsson K. Sense of coherence, social networks, and mental health among children with a cochlear implant. Int J Pediatr Otorhinolaryngol 2015;79(04):610-615

40. Hay-McCutcheon MJ, Hyams A, Yang X, Parton J. Hearing loss and social support in urban and rural communities. Int J Audiol 2018;57(08): 610-617

41. Hay-McCutcheon MJ, Reed PE, Cheimariou S. Positive social interaction and hearing loss in older adults living in rural and urban communities. J Speech Lang Hear Res 2018;61(08): 2138-2145

42. Chapman M, Dammeyer J. The significance of deaf identity for psychological well-being. J Deaf Stud Deaf Educ 2017;22(02):187-194

43. Mousley VL, Chaudoir SR. Deaf stigma: links between stigma and well-being among deaf emerging adults. J Deaf Stud Deaf Educ 2018;23(04): $341-350$
44. Chapman M, Dammeyer J. The relationship between cochlear implants and deaf identity. Am Ann Deaf 2017;162(04):319-332

45. Williams DR, Cooper LA. Reducing racial inequities in health: using what we already know to take action. Int J Environ Res Public Health 2019; 16(04):606

46. Boss EF, Niparko JK, Gaskin DJ, Levinson KL. Socioeconomic disparities for hearing-impaired children in the United States. Laryngoscope 2011;121(04):860-866

47. Barnett M, Hixon B, Okwiri Net al.. Factors involved in access and utilization of adult hearing healthcare: a systematic review. Laryngoscope 2017;127(05):1187-1194

48. Stern RE, Yueh B, Lewis C, Norton S, Sie KC. Recent epidemiology of pediatric cochlear implantation in the United States: disparity among children of different ethnicity and socioeconomic status. Laryngoscope 2005;115(01):125-131

49. Dettman S, Choo D, Dowell R. Barriers to early cochlear implantation. Int J Audiol 2016;55(Suppl 2):S64-S76

50. Kirkham E, Sacks C, Baroody Fet al.. Health disparities in pediatric cochlear implantation: an audiologic perspective. Ear Hear 2009;30(05): 515-525

51. Hernandez DC, Reesor LM, Murillo R. Food insecurity and adult overweight/obesity: gender and race/ethnic disparities. Appetite 2017;117: 373-378

52. Kushalnagar P, Moreland CJ, Simons A, Holcomb T. Communication barrier in family linked to increased risks for food insecurity among deaf people who use American Sign Language. Public Health Nutr 2018;21(05):912-916

53. National Low Income Housing Coalition. Racial Inequities in Housing. Opportunity Starts at Home Accessed June 2021 at: https://www.opportunityhome.org/resources/racial-equity-housing/

54. Wu V, Noel CW, Forner Det al.. Otolaryngology needs among an adult homeless population: a prospective study. J Otolaryngol Head Neck Surg2020;49(01):47

55. Noel CW, Mok F, Wu Vet al.. Hearing loss and hearing needs in an adult homeless population: a prospective cross-sectional study. CMAJ Open 2020;8(01):E199-E204

56. Williams DR, Cooper LA. Reducing racial inequities in health: using what we already know to take action. Int J Environ Res Public Health 2019; 16(04):606

57. Powell W, Jacobs JA, Noble W, Bush ML, SnellRood C. Rural adult perspectives on impact of hearing loss and barriers to care. J Community Health 2019;44(04):668-674

58. D'Agostino EM, Patel HH, Hansen E, Mathew MS, Messiah SE. Longitudinal effects of 
transportation vulnerability on the association between racial/ethnic segregation and youth cardiovascular health. J Racial Ethn Health Disparities 2021;8(03):618-629

59. Sims S, Houston L, Schweinzger I, Samy RN. Closing the gap in cochlear implant access for African-Americans: a story of outreach and collaboration by our cochlear implant program. Curr Opin Otolaryngol Head Neck Surg 2017;25(05): 365-369

60. Billioux A, Verlander K, Anthony S, Alley D. Standardized screening for health-related social needs in clinical settings: the accountable health communities screening tool. NAM Perspectives. 2017;7(05):1-9

61. National Association of Community Health Centers. Protocol for Responding to and Assessing Patients' Assets, Risks, and Experiences (PRAPARE). Accessed June 2021 at: https://www. nachc.org/research-and-data/prapare/

62. American Academy of Family Physicians. The EveryONE Project Toolkit. Accessed June 2021 at: https://www.aafp.org/family-physician/patientcare/the-everyone-project/toolkit.html 